\title{
Search for long-lived particles and other non-conventional signatures at CMS
}

\author{
Alberto Escalante del Valle ${ }^{* \dagger}$ \\ Institute of High Energy Physics, Austrian Academy of Sciences, Vienna, Austria \\ E-mail: a.escalante.del.valleecern.ch
}

\begin{abstract}
Many extensions of the standard model including SUSY predict new particles with long lifetimes, such that the position of their decay is measurably displaced from their production vertex, and particles giving rise to other non-conventional signatures. We present recent results of searches for long-lived particles and other non-conventional signatures obtained using data recorded by the CMS experiment at Run-II of the LHC.
\end{abstract}

The 39th International Conference on High Energy Physics (ICHEP2018)

4-11 July, 2018

Seoul, Korea

* Speaker.

${ }^{\dagger}$ on behalf of the CMS Collaboration. 


\section{Introduction}

Long Lived Particles (LLPs) with sizable lifetimes appear in several Beyond Standard Model (BSM) scenarios in different regions of the model phase space, e.g, mass degenerate states, heavy virtual mediators or small couplings. Recently, the CMS experiment [1] reported new results in searches for LLPs in displaced vertices [2], displaced and emerging jets [3, 4], disappearing tracks [5], as well as heavy stable charged particles [6] and stopped particles in the calorimeters [7]. No signs of LLPs have been reported and results are interpreted in different BSM scenarios.

\section{Displaced vertices, jets and emerging jets}

The decay of LLPs into quarks often leads to jets containing multiple tracks and vertices displaced from the primary interaction vertex $(\mathrm{PV})$. In the displaced vertex search [2], pairs of displaced vertices are built using at least 5 tracks with large impact parameter, $d_{x y} / \sigma_{d_{x y}}>4$ as input, and their distance in the transverse plane is used as discriminant variable. Figure 1 (left) shows one of the interpretations in the context of a long-lived gluino with mass $800 \mathrm{GeV}$ in an R-parity violating Supersymmetric model. The displaced jet search [3] uses an inclusive approach based on a dedicated tagger built using track properties such as their compatibility with the PV, impact parameter distribution or the emission angle. Emerging jets appear in different dark QCD scenarios and contain multiple displaced vertices from the decay of dark hadrons. The emerging jet search [4], targets low mass long-lived dark pions, $\pi_{d}$, formed in the hadronizacion of dark fermions, $\mathrm{Q}_{\mathrm{d}}$, produced in the decay of a dark mediator, $\mathrm{X}_{\mathrm{d}}$, charged under QCD and dark QCD. The search is a generalization of the displaced jet search using an emerging jet tagger optimized for different masses of the dark pion, $m_{\pi_{d}}$, dark mediator, $m_{\mathrm{X}_{\mathrm{d}}}$ and the lifetime of the dark pion, $\tau_{\pi_{d}}$. Figure 1 (right) shows the exclusion curve for a dark QCD model with $m_{\pi_{d}}=10 \mathrm{GeV}$.
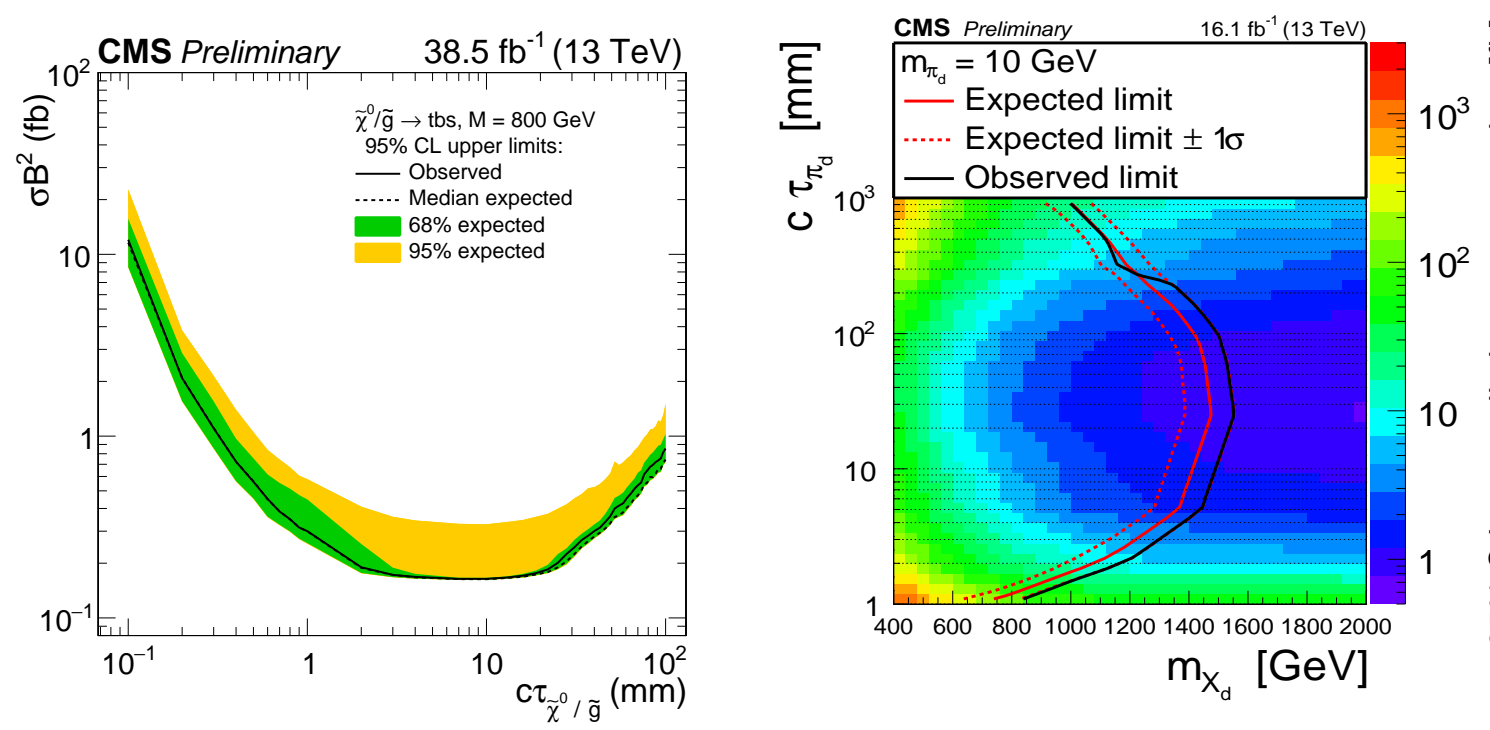

Figure 1: (Left) constraints to a long-lived $\tilde{g} / \tilde{\chi}^{0}$ from the displaced vertices search [2]. (Right) signal exclusion curve for a dark QCD model with $m_{\pi_{d}}=10 \mathrm{GeV}$ from the emerging jets search [4]. 


\section{Disappearing, stopped and heavy stable charged particles}

Disappearing tracks [5] appear in the decay of charged LLPs into objects that are undetected experimentally. The signature in the detector is a reconstructed prompt track with missing hits in the outer layers of the silicon tracker and without corresponding energy deposits in the calorimeters or muon detectors. The search targets an Anomaly Mediated SUSY Breaking (AMSB) model where a chargino, $\tilde{\chi}_{1}^{ \pm}$, is nearly mass degenerate with a neutralino, $\tilde{\chi}_{1}$. As consequence, the chargino is long-lived and the decay products, $\tilde{\chi}_{1}^{ \pm} \rightarrow \tilde{\chi}_{1} \pi^{ \pm}$, are invisible or too soft to be detected. Figure 2 (left) shows the experimental constraints in the chargino mass-lifetime plane. Long-lived heavy stable charged particles (HSCP) with $m_{\mathrm{HSCP}}>100 \mathrm{GeV}$ can travel at non-relativistic speeds, $\beta<$ 0.9, and leave anomalous energy deposits, $\mathrm{dE} / \mathrm{dX}$, in the detector. The HSCP search [6] exploits this striking signature and targets HSCPs with different interactions with the detector to put strong constraints as a function of their mass, $m_{\mathrm{HSCP}}$. LLPs might get trapped in the densest regions of the detector such as calorimeters or the iron yoke, and decay when there are no collisions after long times. The stopped particle search [7] uses a dedicated trigger that selects events at least two bunch crossing away from any proton bunch. One interpretation of the search, shown in Figure 2 (right), is a multiple charged massive particle (MCHAMP) with $m_{\text {MCHAMP }}=400 \mathrm{GeV}$, charge $Q=2 e$ and a lifetime ranging between $100 \mathrm{~ns}$ to 10 days, that decays to a dimuon pair.
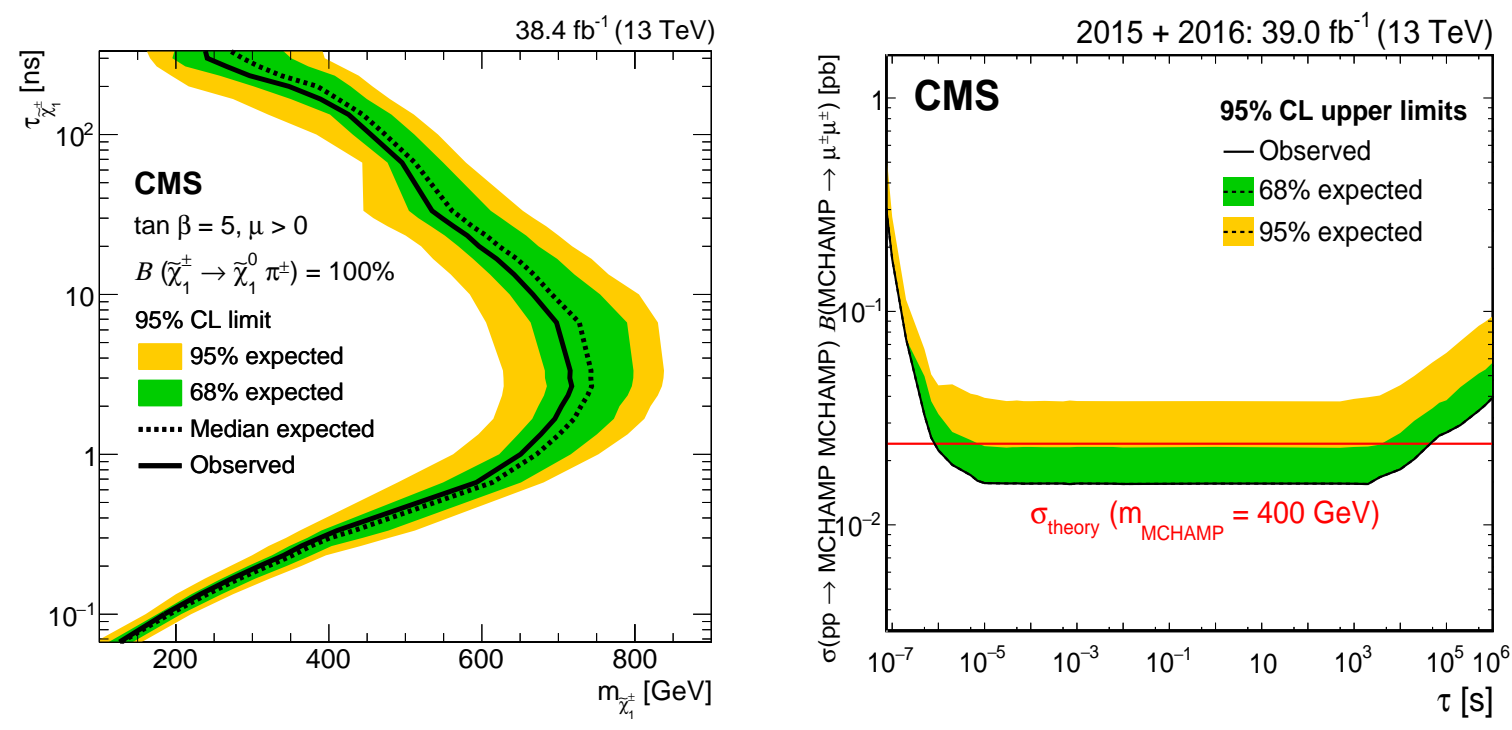

Figure 2: (Left) signal exclusion curve for a chargino in an AMSB model from the disappearing track search [5]. (Right) constraints to a MCHAMP decaying to dimuon pair from the stopped particle search [7].

\section{Reinterpretation of prompt searches}

In addition to displaced decays, due to the probabilistic nature of an LLP, a sizable fraction of its decay products can be prompt or outside of the detector. Studies of the interplay between dedicated and standard searches are important to identify gaps in the experimental coverage. As an example of this, a recent gluino search [8] included a long-lived interpretation. Figure 3 shows that prompt searches relying on tracking start losing sensitivity around $c \tau \approx 1 \mathrm{~cm}$. 


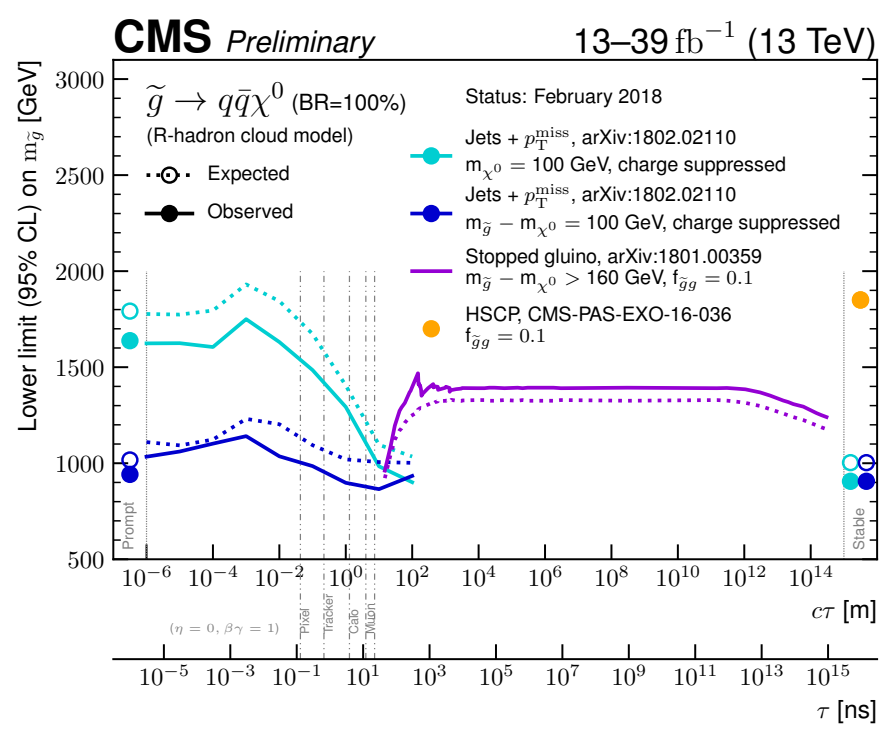

Figure 3: Signal exclusion curves for a long-lived gluino in a split supersymmetry model from prompt and dedicated searches. The prompt curves are from Ref. [8] and the dedicated curves are from Refs. [6,7].

\section{Conclusions}

The talk covered the latest results from CMS in the search for LLPs and focused on displaced vertices, displaced jets, emerging jets, disappearing tracks, heavy stable charged particles and stopped particles. All measurements were consistent with background-only expectations. No signs of LLPs were reported, and constraints in different BSM models were shown.

\section{References}

[1] CMS Collaboration. "The CMS experiment at the CERN LHC. The Compact Muon Solenoid experiment". JINST 3 (2008) S08004.

[2] CMS Collaboration. "Search for long-lived particles with displaced vertices in multijet events in proton-proton collisions at $\sqrt{s}=13 \mathrm{TeV}$ ". CMS-PAS-EXO-17-018, cds.cern.ch/record/2621291.

[3] CMS Collaboration. "Search for new long-lived particles at $\sqrt{s}=13 \mathrm{TeV}$ ". Phys. Lett. B 780 (2018) 432-454.

[4] CMS Collaboration. "Search for new particles decaying to a jet and an emerging jet". CMS-PAS-EXO-18-001, cds.cern.ch/record/2625123.

[5] CMS Collaboration. "Search for disappearing tracks as a signature of new long-lived particles in proton-proton collisions at $\sqrt{s}=13 \mathrm{TeV}$ ". CMS-PAS-EXO-16-044, cds.cern.ch/record/2306201.

[6] CMS Collaboration. "Search for heavy stable charged particles with $12.9 \mathrm{fb}^{-1}$ of 2016 data". CMS-PAS-EXO-16-036, cds.cern.ch/record/2205281.

[7] CMS Collaboration. "Search for decays of stopped exotic long-lived particles produced in proton-proton collisions at $\sqrt{s}=13 \mathrm{TeV}$ ". JHEP 05 (2018) 127.

[8] CMS Collaboration. "Search for natural and split supersymmetry in proton-proton collisions at $\sqrt{s}=$ $13 \mathrm{TeV}$ in final states with jets and missing transverse momentum". JHEP 05 (2018) 025. 